\title{
NOTE ON A PRINCIPAL AXIS TRANSFORMATION FOR NON-HERMITIAN MATRICES
}

\author{
JOHN WILLIAMSON
}

In a recent note the following theorem was proved. $\dagger$

THEOREM 1. If $A$ is a matrix, over the complex field, of $r$ rows and $s$ columns, there exist two unitary matrices $U$ and $V$, such that

$$
U A V=\left(\begin{array}{ll}
D & 0 \\
0 & 0
\end{array}\right)
$$

where $D$ is a real diagonal matrix $\left[d_{1}, d_{2}, \cdots, d_{k}\right]$ with positive elements $d_{i}$.

For completeness the following result may be added: The elements $d_{i}$ are determined uniquely as the positive square roots of the nonzero characteristic roots of the positive hermitian matrix $A A^{*}$, where $A^{*}$ is the conjugate transposed of $A$.

The elements $d_{i}$ thus form a complete set of invariants for the matrix $A$ under such unitary transformations. This, together with the theorem itself, may be proved as follows. If $r \leqq s$ and $k$ is the rank of $A, A=(P, 0) V$, where $V$ is unitary and $P$ is the positive hermitian matrix of order $r$ and rank $k$ uniquely determined $\ddagger$ by the equation $A A^{*}=P^{2}$. The hermitian matrix $P$ is unitarily equivalent to the diagonal matrix of order $r$, whose first $k$ elements are $d_{i},(i=1,2, \cdots, k)$. In case $r \geqq s$, the polar representation

$$
A=U\left(\begin{array}{c}
P \\
0
\end{array}\right)
$$

may be used, to prove the desired result.

The following theorem may also be of interest.

Theorem 2. Let $A$ and $B$ be two matrices, over the complex field, of $r$ rows and s columns. Necessary and sufficient conditions that there exist two unitary matrices $U$ and $V$, such that

$$
U A V=A_{1}, \quad U B V=B_{1},
$$

where $A_{1}$ and $B_{1}$ are diagonal matrices, are that

$\dagger$ Carl Eckart and Gale Young, $A$ principal axis transformation for non-hermitian matrices, this Bulletin, vol. 52 (1939), pp. 118-121.

$\ddagger$ John Williamson, $A$ polar representation of singular matrices, this Bulletin, vol. 41 (1935), pp. 118-123. 


$$
A B^{*}=f\left(B A^{*}\right), \quad B^{*} A=f\left(A^{*} B\right),
$$

where $f(x)$ is a polynomial in $x$.

We first prove the necessity of the conditions. Since $A_{1} B_{1}^{*}$ is a diagonal matrix, $A_{1} B_{1}^{*}$ is normal $\dagger$ and therefore $A_{1} B_{1}^{*}=f\left(B_{1} A_{1}^{*}\right)$. Since $A_{1}$ and $B_{1}^{*}$ are both diagonal matrices, $A_{1} B_{1}^{*}$ coincides with $B_{1}^{*} A_{1}$ except for zero elements and so does $B_{1} A_{1}{ }^{*}$ with $A_{1}^{*} B_{1}$. Therefore $B_{1}^{*} A_{1}=f\left(A_{1}^{*} B_{1}\right)$ and, as a consequence of (1), (2) is true.

To prove the sufficiency we note that there is no loss in generality in assuming that $A$ is of the form

$$
\left(\begin{array}{ll}
D & 0 \\
0 & 0
\end{array}\right)
$$

where $D$ is a real nonsingular diagonal matrix. Let

$$
\left(\begin{array}{ll}
H & L \\
M & K
\end{array}\right)
$$

be a partition of $B$ similar to that of $A$. Since $A B^{*}$ is normal, $A B^{*} B A$ $=B A A B^{*}$ and by a simple calculation we find that

$$
M D D M^{*}=(M D)(M D)^{*}=0 .
$$

Hence $M D=0$ and, since $D$ is nonsingular, $M=0$. In a similar manner by using the fact that $B^{*} A$ is normal we find that $L$ is zero. Conditions (2) now reduce to

$$
D H^{*}=f(H D), \quad H^{*} D=f(D H) .
$$

Since $f(H D)=D^{-1} f(D H) D$, it follows from (3) that $D^{2} H^{*}=H^{*} D^{2}$. But $D$ is a positive definite real matrix. Hence $D H^{*}=H^{*} D$ and consequently $D H=H D$. Since $D H^{*}$ is normal, $H^{*}$ and, therefore $H$, is normal. Since $H$ is normal $\ddagger$ and commutative with $D$, there exists a unitary matrix $U_{1}$, such that $U_{1} D U_{1}^{*}=D$ and $U_{1} H U_{1}^{*}=D_{1}$, where $D_{1}$ is diagonal but not necessarily real. By Theorem 1 there exist two unitary matrices $U_{2}$ and $V_{2}$, such that $U_{2} K V_{2}=D_{2}$, where $D_{2}$ is diagonal and real. If

then

$$
U=\left(\begin{array}{ll}
U_{1} & 0 \\
0 & U_{2}
\end{array}\right), \quad V=\left(\begin{array}{ll}
U_{1}^{*} & 0 \\
0 & V_{2}
\end{array}\right),
$$

$\dagger$ Aurel Wintner, Spektraltheorie der unendlichen Matrizen, Leipzig, 1929, p. 24; John Williamson, Matrices normal with respect to an hermitian matrix, American Journal of Mathematics, vol. 60 (1938), p. 355.

$\ddagger$ Aurel Wintner, op. cit., p. 24. 


$$
U A V=\left(\begin{array}{ll}
D & 0 \\
0 & 0
\end{array}\right), \quad U B V=\left(\begin{array}{ll}
D_{1} & 0 \\
0 & D_{2}
\end{array}\right) .
$$

Since the elements of $D$ and $D_{2}$ are real, we have the following corollary.

Corollary 1. If $k$ is the smaller of the ranks of $A$ and $B, A_{1}$ and $B_{1}$ may be so determined that at most $k$ of the elements of $A_{1}$ and $B_{1}$ are not real.

If $f(x)=x, A B^{*}$ and $B^{*} A$ are both hermitian and $D_{1}$ must be real, so that $B_{1}$ is real. Conversely, if $B_{1}$ is real, $A_{1} B_{1}{ }^{*}$ and $B_{1}{ }^{*} A_{1}$ are both hermitian and therefore $A B^{*}$ and $B^{*} A$ are both hermitian. This result gives Theorem 2 of the note quoted in the first footnote. If $f(x)=1 / x, A B^{*}$ and $B^{*} A$ are both unitary and the elements of $D_{1}$ are $d_{j}^{-1} e^{i \theta j}$.

When $r=s$, so that the matrices $A$ and $B$ are square, condition (2) may be replaced by the condition that $A B^{*}$ be normal and unitarily equivalent to $B^{*} A$. For $A B^{*}$ is similar to $B^{*} A$ and, since both are normal, $A B^{*}$ is unitarily equivalent to $B^{*} A$.

Both Theorems 1 and 2 are true in the real field, if $A^{*}$ denotes the transposed of $A$ and "unitary" is replaced by "orthogonal," except that $D_{1}$ may have two-rowed matrices of the form

$$
\left(\begin{array}{rr}
a & b \\
-b & a
\end{array}\right)
$$

in its diagonal. $\dagger$ In the simplest case, when $A B^{*}$ and $B^{*} A$ are both symmetric, $D_{1}$ will necessarily be diagonal, and Theorem 2 of the paper, quoted in the first footnote, is true in the real field, if "unitary" is replaced by "orthogonal" and "hermitian" by "symmetric."

The Johns Hopkins University

$\dagger$ F. D. Murnaghan and Aurel Wintner, A canonical form for real matrices under orthogonal transformations, Proceedings of the National Academy of Sciences, vol. 17 (1931), pp. 417-420. 